\title{
JUVENTUDE E RESILIÊNCIA: EXPERIÊNCIA COM JOVENS EM SITUAÇÃO DE VULNERABILIDADE
}

\author{
YOUNGSTERS AND RESILIENCE: EXPERIENCE WITH \\ JOUNGSTERS IN VULNERABILITY CONDITION
}

\author{
Karla Rampim Xavier ${ }^{1}$ \\ Silmara Conchão ${ }^{2}$ \\ Nivaldo Carneiro Junior ${ }^{3}$
}

Xavier KR; Conchão S; Carneiro Junior N. Juventude e resiliência: uma experiência com jovens em situação de vulnerabilidade. Rev. Bras. Cresc. e Desenv. Hum. 2011; 21(1): 140-145.

\section{Resumo:}

O objetivo deste trabalho é identificar estratégias para ações que diminuam situações de vulnerabilidades entre jovens. Assim, este artigo apresenta resultados da pesquisa com jovens em situação de vulnerabilidade social na cidade de Santo André, São Paulo, visando à identificação de estratégias para ações nas áreas sociais, que contribuam com a diminuição das suas vulnerabilidades, empregando o conceito de resiliência. Através do método qualitativo, utilizou-se a estratégia de grupos de conversa, guiados por temas: 1) "contextualização do projeto e discussão sobre o conceito de resiliência”; 2) "minha situação atual e meus propósitos”; 3) "minhas capacidades e recursos” e 4) "recuperar as fortalezas”. Participaram 11 jovens de idade entre 15 a 19 anos, inseridos nos diversos Programas Sociais da Prefeitura de Santo André. Os grupos foram gravados em áudio, transcritos empregando análise de conteúdo. Esse trabalho foi desenvolvido no período de 30 de novembro a 02 de dezembro de 2007. Os jovens ao relatarem suas experiências de exposição à violência doméstica, relação com drogas, conflito com a lei, rejeição familiar e social, entre outras, reelaboraram sua própria história e perceberam suas potencialidades de superação, associando-se, nesse momento, o conceito de resiliência e fortalecimento de suas competências. Tal estratégia possibilitou a reflexão e a exposição dos seus sonhos e desejos como uma possibilidade. A metodologia utilizada permite aproximação e resultados mais efetivos com grupo-alvo, sendo, portanto, promissora para os programas e as ações dirigidos a eles por agentes públicos.

Palavras-chave: juventude; vulnerabilidade; resiliência; programas sociais.

\footnotetext{
Psicóloga, psicanalista, Prefeitura de Diadema, São Paulo, karla.rampim@gmail.com

Mestre em sociologia, professora da Faculdade de Medicina do ABC, Santo André, São Paulo, saconchao@uol.com.br

Doutor em medicina preventiva, professor da Faculdade de Medicina do ABC, Santo André, São Paulo, nicarneirojr@uol.com.br Endereço para correspondência: Faculdade de Medicina do ABC. Av. Príncipe de Gales, nº 821 - Vila Príncipe de Gales Santo André - São Paulo - SP - CEP: 09060-870. E-mail: secsaudecoletiva@fmabc.br

Artigo baseado nos resultados do projeto 'La prevención de la exclusión social en jóvenes y adolescentes en contextos urbanos mediante la promoción de la salud y de los factores resilientes”. Programa URB-AL, Red 10, “Lucha contra la Pobresa”, com apoio técnico e financeiro da Comunidade Européia, desenvolvido em Santo André (Brasil), Montevideo e Salto (Uruguai), Medelin (Colômbia), l’Alt Empordà (Espanha) e Trieste (Itália).
} 


\begin{abstract}
:
The objective is to identify strategies for actions to reduce situations of vulnerability among young. This article presents results of a job carried out with youngsters in a social vulnerability condition at the city of Santo André, São Paulo, aiming to identify strategies towards social actions which can contribute for the reduction in their vulnerabities, using the resilience concept. Through a qualitative methodology, it was adopted a strategy of chatting groups, guided by subjects: 1) "project context and discussion about the resilience concept"; 2) "my current situation and my intentions"; 3) "my capacities and resources" 4) "to recover the strenght". We counted on 11 young people, between 15 and and 19 years old who were included in several kinds of Social Programs maintained by Santo Andrés city hall. The discussions had been recorded in audio, transcribed and analyzed. This job was developed from November 30th up to December 02nd, 2007. The youngsters talked about their experiences on domestic violence, drugs, law conflict and familiar and social rejection, elaborating once more their own histories and realizing their overcoming potentialities, associating, at this moment, the resilience concept and the strengthening of their own competencies. Such strategy made possible the reflexion and exposition of their dreams and wishes as a real possibility. The chosen methodology allows more effectives approach and effective results with the target-group, and therefore it is promising to be used by social programs and actions focused on them.
\end{abstract}

Key words: youth; vulnerability; resilience, social programs.

\section{INTRODUÇÃO}

Juventude agrega diversas interpretações pela própria condição intermediária entre a criança e o adulto e, muitas vezes, é tomada como sinônimo de adolescente. É fundamental considerar que os conteúdos, a duração e a atribuição de significados sociais aos diferentes momentos de vida são culturais e históricos. Nesse sentido, infância, adolescência, juventude, idade adulta e velhice são construções socio-históricas ${ }^{1,2}$.

Há certo consenso em considerar como jovem o grupo etário de 15 a 24 anos, reconhecendo dois subgrupos de idades com características distintas socioculturalmente: 15 a 19 anos e 20 a 24 anos, sendo o primeiro reconhecido como adolescente ${ }^{3}$. No Brasil, o Estatuto da Criança e do Adolescente (ECA) ${ }^{4}$ considera a faixa de 12 a 18 anos como adolescente.

De acordo com Abramo 5 , podemos supor que a juventude seja um barômetro das mudanças sociais, pois a reconhecemos como uma geração responsável pela transmissão de valores ou pela ruptura de determinados padrões. Desse modo, ela pode ser tomada como responsável pela transgressão de mecanismos que presidem a integração social. Mais que isso, ela é usualmente analisada a partir da ótica de "problema social" quando se afasta das expectativas sociais nela depositadas ${ }^{6}$.

Promover o desenvolvimento saudável da juventude é um dos mais importantes investimentos que uma sociedade pode fazer. Nesse sentido, o reconhecimento e a valorização das características individuais facilitadoras para o envolvimento efetivo no desenvolvimento de ações orientadas para a promoção à saúde são fundamentais, ainda mais em nossa sociedade com padrões de desigualdades sociais marcantes, ocasionando inserções sociais precárias, particularmente entre os jovens, que crescem em situações de adversidades, que limitam seu pleno desenvolvimento, necessitando de esforços individuais e apoios externos para superar essas condições?

Nesse cenário, um instrumento conceitual que se tem trabalhado na abordagem des- 
sas situações e contribuído para formulação de ações é o conceito de resiliência, que segundo Bernard" é "a capacidade de um indivíduo de reagir diante as adversidades, o que implica um conjunto de qualidades que fomentam um processo de adaptação exitosa e de transformação, apesar dos riscos e da própria adversidade vivida de exclusão social”.

$\mathrm{O}$ termo resiliência tem sua origem do latim, resilio, que significa voltar atrás. A psicologia apropria-se desse conceito e redefine como a capacidade de o indivíduo, ou a família, enfrentar as adversidades, ser atravessado por elas, mas, conseguir superá-las. Portanto, resiliência não pode ser entendida como uma qualidade inata ou que se adquire naturalmente durante a vida. Ela deve ser entendida como um ganho adquirido num processo interativo do sujeito e sua inserção no meio social ${ }^{9,10,11}$.

Santo André é uma das sete cidades que compõem a Região do ABC, localizada na Região Metropolitana de São Paulo, com aproximadamente 650.000 habitantes. De acordo com o Mapa da Juventude de Santo André (2006) a cidade possuía 121.805 jovens de 15 a 24 anos. A grande maioria desses jovens era alfabetizada (98,9\%). Porém, 49,2\% não frequentavam a escola. Dentre os 60.918 jovens (50,8\%) que estudavam, 65,1\% estavam na rede pública e $34,9 \%$ na rede particular de ensino. A taxa de desemprego nesse grupo etário encontrava-se em torno de 32,6\%. O que se pode observar sumariamente a partir desses dados é uma inserção precária desses jovens, particularmente em relação à educação e ao mercado de trabalho ${ }^{12,13}$.

O desenvolvimento desta pesquisa com os jovens em Santo André no contexto do projeto URB-AL ${ }^{14}$, centrando-se na categoria analítica de resiliência, objetiva, particularmente, reconhecer as estratégias pessoais e coletivas de superação em condições sociais desfavoráveis ao desenvolvimento humano e social; implicar os jovens; trabalhar a autoconfiança dos jovens e suas pos- sibilidades de desenvolvimento pessoal e organizar objetivos de vida saudáveis. Assim, o objetivo deste trabalho é identificar estratégias para ações que diminuam situações de vulnerabilidades entre jovens.

\section{MÉTODO}

O método utilizado situa-se no campo das pesquisas qualitativas, apoiando-se nos pressupostos da pesquisa-ação ${ }^{15}$. A estratégia de rodas de conversa temáticas (ou grupos de conversa) foi adotada neste trabalho.

Foram realizadas quatro rodas de conversa, com os seguintes temas: 1) "contextualização do projeto e discussão sobre o conceito de resiliência"; 2) "minha situação atual e meus propósitos"; 3) "minhas capacidades e recursos” e 4) “recuperar as fortalezas”. Esses grupos tiveram duração média de três horas e foram intercalados com atividades lúdicas. As rodas de conversa foram gravadas em meio digital, como também feitas anotações em diário de campo.

Esse trabalho foi desenvolvido no período de 30 de novembro a 02 de dezembro de 2007, na Vila de Paranapiacaba, Santo André/São Paulo. O Centro de Formação Sócio Ambiental foi o local de realização das atividades, como também de hospedagem dos participantes.

A equipe de coordenação foi composta pelos autores deste artigo, com apoio de um cineasta e um arte-educador musical.

Participaram 11 jovens de idade entre 15 a 19 anos, nove garotos e duas garotas. Esses jovens foram indicados pelos "Programas Sociais” da Prefeitura de Santo André - Casa de Acolhida, ProJovem, Redução de Danos, Liberdade Assistida e Programa de Jovens de Paranapiacaba -, de acordo com suas histórias de vida, suas capacidades de liderança e seus desempenhos em busca de autonomia.

Foi realizada com cada jovem partici- 
pante uma entrevista não estruturada, gravada, visando um diagnóstico inicial das suas experiências de vida.

Nos grupos, frases norteadoras para o desenvolvimento da reflexão foram apresentadas: "Eu tenho - fatores de superar/suportar o externo"; "Eu sou - forte”; "Eu posso - habilidades pessoais"; "Eu estou - o que estou disposto a fazer".

Trabalhar essas questões no grupo promove a exteriorização dos sentimentos, a escuta de si e a escuta do outro, como também propicia a criação de vínculo e a interação entre os seus participantes.

A análise de conteúdo foi empregada em todo material das transcrições e das anotações das rodas de conversa.

Esse projeto de pesquisa foi apreciado pelo Comitê de Ética em Pesquisa da Secretaria de Saúde de Santo André. Todos os jovens participantes, assim como seus familiares ou responsáveis, foram informados sobre os objetivos e os procedimentos metodológicos do projeto, assinando o Termo de Consentimento Livre e Esclarecido.

\section{RESULTADOS E DISCUSSÃO}

Os jovens participantes, de modo geral, relataram experiências de vida reveladoras de uma série de adversidades, tais como: exposição à violência doméstica, estreita relação com as drogas, conflito com a lei, rejeição no âmbito familiar e social, experiências de abandono, preconceito e grande vulnerabilidade econômica:

“...você não viu os meus dentes... é de fumar crack” (jovem, sexo masculino, 16 anos);

“...antes mesmo de nascer, meu vô já não gostava de mim... Ele expulsou minha mãe de casa, quando ela estava grávida de mim” (jovem, sexo masculino, 16 anos);

“...tô cumprindo medida porque fui la- ranja num assalto de carro" (jovem, sexo masculino, 18 anos).

No momento em que puderam falar das suas vivências, se escutaram ao mesmo tempo. Despertou-se ali o reconhecimento das suas próprias histórias, possibilitando a percepção de suas potencialidades em tais situações. Enquanto contavam suas experiências, tiveram a oportunidade de reelaborar as situações vividas.

Tal descoberta significou, em boa medida, fazer com que esses jovens se tornassem mais confiantes e resilientes, pois, apesar de tantas dificuldades na interação com a sociedade, na dinâmica foi permitindo a construção de perspectivas futuras. E no momento em que foi proposta a discussão em grupo, de uma forma mais lúdica, além de conhecerem várias outras experiências, visualizaram projetos de vida para os próximos cinco anos, incluindo seus sonhos e desejos nessa projeção:

"eu pretendo fazer um curso de teatro, pra fazer uma boa faculdade de ator" (jovem, sexo masculino, 16 anos);

“...trabalhar fora do Brasil em hotéis" (jovem, sexo masculino, 15 anos).

Importante ressaltar que não houve a eliminação do problema, mas uma ressignificação e o reconhecimento da forma como ele próprio conseguiu passar por seus momentos de adversidades. Estava ali se revelando e ao mesmo tempo se percebendo como sujeito e autor de sua própria história:

“...nossa!!... Eu nunca tinha falado isso com ninguém antes” (jovem, sexo masculino, 16 anos).

Dessa forma, durante o trabalho com esses jovens tais estratégias e práticas possibilitaram que, a partir da "reelaboração" de suas vidas, eles pudessem refletir e falar dos seus sonhos e desejos como uma possibilidade real de serem vivenciados:

"quero fazer faculdade, ser uma cantora, ter minha casa, trabalhar na área de rádio ou TV. Ter uma renda boa... Ser feliz. Ter via- 
jado para alguns lugares” (jovem, sexo feminino, 16 anos);

"vou fazer computação e fazer curso para cuidar dos animais, ser veterinário" (jovem, sexo masculino, 16 anos).

Os jovens se envolveram com todas as atividades desenvolvidas de forma responsável e integral:

"tudo foi muito bom para mim. Os debates sobre resiliência, a oficina de filmagem e de fotografia, ritmo e poesia, os passeios, os integrantes do grupo, o tango..." (jovem, sexo masculino, 19 anos).

Refletiram sobre suas vidas no contexto da realidade social; tristezas, medos, sonhos, desejos, esperanças e perspectivas foram expostos e problematizados. O significado sobre resiliência foi apresentado e apreendido no grupo.

Durante a apresentação do conceito o grupo foi questionado se conhecia alguém resiliente e um dos jovens (sexo masculino, 19 anos) contou a história do seu primo, e posteriormente, contou sua própria experiência de abandono aos oito anos de idade e finalizou a sua fala: "eu sou um resiliente, resiliente para sempre”.

Os grupos promoveram a reflexão individual e coletiva sobre a perspectiva de vida, o autoconhecimento, a interação entre os pares, a identificação da experiência com outras histórias de vida, entre outros, construindo, desse modo, elementos de forte interação e possibilidade de sociabilidades:

“...foi bom conhecer pessoas novas, trocar experiências, vários desafios enfrentados, as pessoas que conhecemos são dez... Espero ter outro encontro..." (jovem, sexo masculino, 15 anos);

“...tudo foi muito bom, principalmente a união entre nós” (jovem, sexo masculino, 18 anos).

Ao privilegiar os temas sonho, desejo e perspectiva de vida, a metodologia provocou situações em que esses jovens perceberam o seu potencial, acreditaram mais na sua capacidade de ser e de agir, fortalecendo assim a autoestima, o autocuidado, a autonomia e convidando-os em todos os momentos a se identificarem como sujeitos protagonistas das suas histórias, podendo desta forma, afastá-los da exposição aos riscos, através da capacidade de planejar e perseguir projetos futuros para uma melhor qualidade de vida, possibilitando impactos positivos sobre sua própria saúde e a saúde da comunidade na qual estão inseridos:

“...quero estar com meus objetivos, $C D$ completo e gravado... Com a vida normal, com a minha família e lutando sempre para ser feliz” (jovem, sexo masculino, 18 anos).

Pode-se perceber que, no contexto deste trabalho, a utilização do conceito de resiliência adquire significado importante no desenho de estratégias de intervenção com segmentos populacionais em situação de vulnerabilidade social.

Traz, portanto, novos recursos potencializadores para a formulação de atitudes que contribuam para o fortalecimento e o desenvolvimento de competências nos indivíduos implicados de decisões promotora da saúde.

Ao se utilizar, também, a escolha privilegiada da estratégia metodológica empregada no desenvolvimento desse trabalho, permitiu uma aproximação com esses jovens, revelando-se extremamente apropriada para a abordagem com o grupo-alvo em diferentes espaços e práticas sociais.

Em reunião de avaliação com agentes públicos, responsáveis pelos Programas Sociais nos quais esses jovens estavam inseridos, foram perceptíveis a mudança de atitudes e participação deles nessas organizações.

Pode-se, nesse sentido, exemplificar a participação de um desses jovens, que se integrou ao trabalho da equipe de saúde da juventude da Secretaria de Saúde de Santo André, sendo "multiplicador de boas práticas de saúde" entre diversos grupos juvenis da cidade, como também, participou ativa- 
mente na articulação juvenil para a formulação do Plano Municipal de Saúde da Juventude da cidade.

A necessidade de ampliar, avaliar e consolidar essa experiência se coloca para os 7 programas e ações destinados aos jovens, par-

\section{REFERÊNCIAS}

1. Araújo TW, Calazans G. Adolescência, vulnerabilidade e Sexualidade. In: Araújo TW, Calazans G (orgs.). Prevenção das DST/ AIDS em adolescentes e jovens: Brochuras de referência para os profissionais de saúde. São Paulo. 2007, p. 9-24.

2. Pimenta MM. "Ser jovem e ser adulto”: identidades, representações e trajetórias [tese]. São Paulo Faculdade de Filosofia, Letras e Ciência Humanas da Universidade de São Paulo; 2007. p. 464.

3. Organização Mundial da Saaúde (OMS). Young Peoples Health: a challenge for society. Relatório do Grupo de Trabalho sobre Jovens e Saúde para Todos no Ano 2000. Série de Relatórios Técnicos: Genebra, 1986, p. 87-127.

4. Brasil. Lei n. 8069 de 13 de julho de 1990. Institui o Estatuto dos Direitos da Criança e do Adolescente - ECA. Brasília; 1990 [acesso em 03 jul 2010]. Disponível em: www.planalto.gov.br/ccivil_03/Leis/ L8069.html

5. Abramo HW. Considerações sobre a temática social da juventude no Brasil. Coleção Educação para Todos. In: Fávero O, Spósito MP, Carrano P, Novaes RR (orgs.). Juventude e Contemporaneidade. Coleção Educação para Todos. Ministério da Educação (MEC), Organização das Nações Unidas para Educação, ciência e cultura (UNESCO); 2007. p. 73-92.

6. Zioni F. A questão social na França contemporânea: uma experiência de pesquisa. Rev Bras Crescimento Desenvolvimento Hum. 2009; 19(2): 249-261.

7. Buss PM. Uma introdução ao conceito de promoção da saúde. In: Czeresnia D, Freitas ticularmente aquelas em situações de vulnerabilidades sociais, pautando essa questão para os agentes públicos nas diversas áreas. É o que se aponta a partir deste trabalho, como também no contexto geral do Projeto URB-AL.

CM, (orgs.). Promoção da saúde: conceitos, reflexões, tendências. Rio de Janeiro; Editora Fiocruz, 2003. p. 15-38.

8. URB-AL. Oficina de Cooperación. Documento de Trabajo - Guía de conceptos y pautas de intervención para La incorporación de estratégias de promoción para la salud y de promoción de La resiliencia en adolescentes. Gabinet d'Estudis Socials. Barcelona, 2006. p. 79.

9. Silva NC, Cardoso PS, Moraes TNP, Centa ML. Resiliência: nova perspectiva na Promoção da Saúde da Família? - Ciência \& Saúde Coletiva (ABRASCO). Rio de Janeiro. 2009; 14(2):497-506.

10. Junqueira MFPS, Deslandes SF. Resiliência e maus-tratos à criança - Caderno de Saúde Pública. Rio de Janeiro. 2003; 19(1):227235.

11. Tavares JA. Resiliência na sociedade emergente. São Paulo; Cortez, 2001.

12. Santo André. Prefeitura de Santo André. Secretaria de Orçamento e Planejamento Participativo - Departamento de Indicadores Sociais e Econômicos. Sumário de Dados 2006 - Ano base: 2005. São Paulo, 2006.

13. Soares CB, Campos CMS. (coord.). Mapa da Juventude de Santo André. Faculdade de Medicina, Universidade de São Paulo. São Paulo, 2007. p. 27.

14. URB-AL. Oficina de Cooperación. Documento de Trabajo - Propuesta de estudio diagnostico sobre la exclusión social de jóvenes y adolescentes. Gabinet d'Estudis Socials, Barcelona, 2006. p. 05.

15. Meyer J. Usando métodos qualitativos na pesquisa-ação relacionada à saúde. In: Pope C, Mays N (orgs.). Pesquisa qualitativa na atenção à saúde. Trad. Ananyr Porto Fajardo. $2^{\mathrm{a}}$ Ed. Porto Alegre; Artmed, 2005. pp. 71-86.

Recebido em: 16/abr./10

Modificado em: 22/out./10 Aceito em: 30/dez./10 